\title{
Vaginal Cancer: About 16 Cases
}

\author{
R Briki, M Makni, K Ouazzani Taibi, S Ernez, E Gana, C Chelly, S Boughizane, H Khairi \\ Department of Gynecology and Obstetrics, University Hospital Farhat Hached, Sousse, Tunisia
}

\section{INTRODUCTION}

- Vaginal cancer is one of the rarest gynaecological cancers. - Primary cancer of the vagina comprises approximately $3 \%$ of all malignant neoplasms of the female genital tract.

- Most of these tumors are squamous cell carcinomas, but melanoma, sarcoma, adenocarcinoma, and other histologic types also occur.

- Although primary vaginal cancer is rare, metastatic disease to the vagina or local extension from adjacent gynecologic structures is not uncommon. As a result, the majority of vaginal malignancies are metastatic, often arising from the endometrium, cervix, vulva, ovary, breast, rectum, and kidney [1].

- Vaginal metastases may occur by direct extension (eg: cervix, vulva, endometrium) or by lymphatic or hematogenous spread (eg: breast, ovary, kidney).

\section{OBJECTIVES}

$\checkmark$ Describe the epidemiological aspects and clinical manifestations of vaginal cancer.

$\checkmark$ Specify the histological aspects as well as histopathological techniques leading to an accurate diagnosis.

$\checkmark$ Study the evolution and prognosis of these tumors after treatment.

\section{METHODS}

This is a retrospective and descriptive study of all cases of vaginal cancer listed from January 2004 through December 2017, diagnosed both at the Department of Gynecology and Obstetrics and the pathology laboratory of the University Hospital Farhat Hached in Sousse, Tunisia.

\section{RESULTS}

- 16 cases were collected.

- Average age was 56.12 years [range $22-75$ ].

- Median age was 65 , and $56 \%$ were over 60 years old.

- Discovery symtomatology was dominated by painless vaginal bleeding that's not associated with menstruation, and smelly vaginal discharge (Table I)

Table I: Clinical manifestations of vaginal cancer.

\begin{tabular}{lc}
\hline Symptoms & Number \\
\hline Abnormal vaginal bleeding & 5 \\
\hline Bleeding after sexual intercourse & 3 \\
Smelly vaginal discharge & 4 \\
Pain when urinating & 2 \\
Pelvic pain & 3 \\
\hline
\end{tabular}

- Majority of vaginal cancers reported were squamous cell carcinomas $(n=7)$, followed by adenocarcinoma $(n=5)$.

- The most FIGO stage was IV $(n=7)$.

- For treatment:

- Radiotherapy in 15 cases
- Concomitant chemotherapy and radiotherapy in one case
Colpohysterectomy and abdominal perineal amputation in 2
[1] Siegel RL, Miller KD, Jemal A. CA Cancer J Clin. 2015.
[2] Shah CA, et al. Obstet Gynecol. 2009.
[3] Gadducci A, et al. Crit Rev Oncol Hematol. 2015.
[4] Alemany L, et al. Eur J Cancer 2014.

Overall survival (OS) at 5 years was function of the initial tumor extension:

$$
\begin{aligned}
& \rightarrow \text { It was on average } 60 \% \text { for } \mathrm{T} 1 \text { or } \mathrm{T} 2 \text { tumors, } \\
& \rightarrow 28 \% \text { for } \mathrm{T} 3 \text { or } \mathrm{T} 4 \text {. }
\end{aligned}
$$

But, it is important to correlate these statistics with the advanced age of patients.

\section{DISCUSSION}

\section{Epidemiology and Risk Factors}

- Approximately 1 in 100,000 women will be diagnosed with in situ or invasive vaginal cancer (typically of squamous cell histology) [2, 3].

- The mean age at diagnosis of squamous cell carcinoma, the most common histologic type of vaginal cancer, is approximately 60 years, although the disease is seen occasionally in women in their 20 s and 30 s. Squamous carcinoma is more common as the age of the patient increases [2].

- Most cases of vaginal cancer are likely mediated by human papillomavirus (HPV) infection, as with cervical cancer [4]. In a case-control study of 156 women with in situ or invasive vaginal cancer, over 50 percent were positive for antibodies to HPV subtypes 16 or 18 [5]. Thus, vaginal cancer has the same risk factors as cervical neoplasia: multiple lifetime sexual partners, early age at first intercourse, and being a current smoker $[5,6]$. - Some research suggests that using a pessary to treat a prolapsed uterus may, over time, irritate the vagina and potentially cause vaginal cell changes.

\section{Diagnosis of Vaginal Cancer}

It requires pathological confirmation by Biopsy of the affected tissue, which may need to be performed under anaesthetic.

\section{Management of Vaginal Cancer}

- Treatment depends on staging work-up.

- Vaginal cancer is staged by FIGO system of staging and TNM staging.

- There are many prognostic factors influencing the choice of treatment. Lymph node metastasis is one of the important prognostic factors, others to mention are histology, size, age. In a recent SEER analysis of over 2000 patients, the 5 year disease specific survival was $84 \%$ for stage $1,75 \%$ for stage II and $57 \%$ for advanced tumors.

- Early carcinomas are generally treated with either surgery or radiation therapy. Advanced cancers are treated with radiation therapy with simultaneous administration of combined chemotherapy.

\section{Preventive Strategies}

Include safe sex and HPV vaccination.

\section{CONCLUSION}

Primary vaginal cancer should be carefully assigned as spread from cervix, vulva, and other metastatic tumors to vagina can occur.

The incidence of high-risk HPV-induced cancers is increasing in younger women.

The FIGO staging of vaginal cancer (2009) follows the same rules as cervical cancer; it is clinically staged and allows the use of routine investigative modalities for staging. Although FIGO encourages the use of advanced imaging modalities, such as computed tomography, magnetic resonance imaging (MRI), and positron emission tomography (PET), to guide therapy. 